\title{
Prime Focus Spectrograph (PFS): the prime focus instrument
}

Wang, Shiang-Yu, Huang, Pin-Jie, Chen, Hsin-Yo, Kimura, Masahiko, Wen, Chih-Yi, et al.

Shiang-Yu Wang, Pin-Jie Huang, Hsin-Yo Chen, Masahiko Kimura, Chih-Yi Wen, Chi-Hung Yan, Jennifer Karr, Richard C. Y. Chou, Yin-Chang Chang, Shu-Fu Hsu, Yen-Shan Hu, Hung-Hsu Ling, Dan J. Reiley, Mitsuko Roberts, James E. Gunn, Craig Loomis, Robert H. Lupton, Hassan Siddiqui, Graham J. Murray, Decio Ferreira, Leandro Henrique dos Santos, Ligia Souza Oliveira, Antonio Cesar de Oliveira, Lucas Souza Marrara, Naoyuki Tamura, Yuki Moritani, Naruhisa Takato, "Prime Focus Spectrograph (PFS): the prime focus instrument," Proc. SPIE 11447, Ground-based and Airborne Instrumentation for Astronomy VIII, 114477V (13 December 2020); doi: 10.1117/12.2561194 


\title{
Prime Focus Spectrograph (PFS): the Prime Focus Instrument
}

\author{
Shiang-Yu Wang*a, Pin-Jie Huang ${ }^{\mathrm{a}}$, Hsin-Yo Chen ${ }^{\mathrm{a}}$, Masahiko Kimura ${ }^{\mathrm{a}}$, Chih-Yi Wen ${ }^{\mathrm{a}}$, Chi-Hung \\ Yan $^{a}$, Jennifer Karr ${ }^{a}$, Richard C. Y. Chou ${ }^{a}$, Yin-Chang, Chang ${ }^{a}$, Shu-Fu Hsu ${ }^{a}$, Yen-Sang Hu ${ }^{a}$, Hung- \\ Hsu Ling ${ }^{\mathrm{a}}$, Dan J. Reiley ${ }^{\mathrm{b}}$, Mitsuko Roberts ${ }^{\mathrm{b}}$, James E. Gunn ${ }^{\mathrm{c}}$, Craig Loomis ${ }^{\mathrm{c}}$, Robert H. Lupton ${ }^{\mathrm{c}}$, \\ Hassan Siddiqui $^{\mathrm{C}}$, Graham J. Murray ${ }^{\mathrm{d}}$, Decio Ferreira ${ }^{\mathrm{e}}$, Leandro Henrique dos Santos ${ }^{\mathrm{e}}$, Ligia Souza \\ Oliveira $^{\mathrm{e}}$, Antonio Cesar de Oliveira ${ }^{\mathrm{e}}$ and Lucas Souza Marrara ${ }^{\mathrm{e}}$, Naoyuki Tamura ${ }^{\mathrm{f}}$, Yuki Moritani ${ }^{\mathrm{f}}$, \\ Naruhisa Takato ${ }^{g}$ \\ anstitute of Astronomy and Astrophysics, Academia Sinica, P. O. Box 23-141, Taipei, Taiwan. \\ ${ }^{\mathrm{b} C}$ California Institute of Technology, 1200 E California Blvd, Pasadena, CA 91125, USA; \\ ${ }^{c}$ Princeton University, Princeton, New Jersey, 08544, USA; \\ dDurham University, Durham, DH1 3LE, UK; \\ eLaboratório Nacional de Astrofísica, Itajubá, 37504-364 Minas Gerais, Brazil; \\ ${ }^{\mathrm{f}}$ Kavli Institute for the Physics and Mathematics of the Universe(WPI), The University of Tokyo, \\ gSubaru Telescope, National Astronomical Observatory of Japan, 650 North Aohoku Place, Hilo, \\ Hawaii, USA.
}

\begin{abstract}
The Prime Focus Spectrograph (PFS) is a new optical/near-infrared multi-fiber spectrograph design for the prime focus of the $8.2 \mathrm{~m}$ Subaru telescope. PFS will cover 1.3 degrees diameter field with 2394 fibers to complement the imaging capability of Hyper SuprimeCam (HSC). The prime focus unit of PFS called Prime Focus Instrument (PFI) provides the interface with the top structure of Subaru telescope and also accommodates the optical bench in which Cobra fiber positioners and fiducial fibers are located. In addition, the acquisition and guiding cameras (AGCs), the cable wrapper, the fiducial fiber illuminator, and viewer, the field element, and the telemetry system are located inside the PFI. The mechanical structure of the PFI was designed with special care such that its deflections sufficiently match those of the HSC's Wide Field Corrector (WFC) so the fibers will stay on targets over the course of the observations within the required accuracy. The delivery of PFI components started in 2017. After the verification of these components, the mechanical structure of the PFI is fully assembled in early 2019 and all Cobra positioners are integrated in summer 2020. A temperature controlled chamber with precise $x-y$ scanner was setup for the verification of the Cobra fiber positioners. The testing of the target convergence performance of Cobra positioners is now in progress.
\end{abstract}

Keywords: Prime Focus, mechanical structure, guiding camera, multi-fiber, spectrograph

\section{INTRODUCTION}

The PFS ${ }^{1}$ is a new multi-fiber spectrograph for the Subaru telescope and it will provide low to medium resolution spectrum for the scientific objects from $0.38 \mu \mathrm{m}$ to $1.26 \mu \mathrm{m}$. The PFS shares the same WFC with the HSC ${ }^{2}$ which is a new wide field camera with 1.5 degrees field of view (FoV). The 2394 fibers populate in a hexagon shape on the prime focal plane of Subaru telescope covering 1.3 degrees diameter field. Each fiber is driven by a Cobra positioner which has two miniature rotational motors to provide two degrees of freedom in a $9.5 \mathrm{~mm}$ diameter patrol region on the focal plane. The PFI is the prime focus unit of PFS to be installed in the prime focus structure, called POpt2, of the Subaru telescope. The primary functions of the PFI is to provide the mechanical interface for the Cobra optical bench (COB) where the science fiber positioners and fixed fiducial fibers are mounted and support the science fiber routing from the focal plane to the spectrographs off the telescope. In addition, the PFI also includes AGCs, the field center camera, the cable wrapper, the field element, the fiducial fiber illuminator and viewing camera, control electronics, and the telemetry system inside the PFI. The flat fielding and spectrum calibration lamps are also positioned on the top of PFI.

* sywang@asiaa.sinica.edu.tw; phone 8862 2366-5338; fax 8862 2367-7849; www.asiaa.sinica.edu.tw 
Being at the prime focus environment of Subaru telescope, tight space, weight and heat dissipation constraints are applied to PFI. Furthermore, structure stiffness of the PFI is also limited to avoid any possible damage of the fragile ceramic lens barrel of the wide field corrector. A combination of different materials at different locations in height is used in PFI to provide a stable focal plane position over the operation temperature range $\left(5\right.$ to $\left.-5{ }^{\circ} \mathrm{C}\right)$ of PFS. A glycol based cooling system removes the heat generated from the electronics of various components of PFI to avoid possible seeing degradation. To share the same wide field corrector with HSC, a flat glass called field element is added in front of the fibers for compensating the optical path difference of the HSC filter and window.

Six FLI ML4720 cameras are installed at the periphery of the effective FoV for field acquisition and guiding. A $0.9 \mathrm{~mm}$ BK7 glass is installed inside each camera to cover half of the CCD sensor to accelerate the focus sequence. Together with a tiny $0.75 \mathrm{~mm}$ viewing camera at the field center, AGCs will provide essential information for the pointing and distortion map of the focal plane. The relative distance between AGC sensors and fiducial fibers will be calibrated under different operation conditions and kept stable within 5 microns during a exposure to meet the alignment requirement of the fibers. A high brightness red LED with a light diffusor is used as the light source to illuminate 96 fiducial fibers.

The PFS collaboration is led by Kavli Institute for the Physics and Mathematics of the Universe, the University of Tokyo with international partners consisting of Academia Sinica, Institute of Astronomy and Astrophysics in Taiwan, Caltech/Jet Propulsion Laboratory, Princeton University/John Hopkins University and NorthEast Participation Group in USA, Chinese PFS Participating Consortium in China, Laboratoire d'Astrophysique de Marseille in France, Max Planck Institute for Astronomy in Germany, National Astronomical Observatory of Japan/Subaru Telescope, and Universidade de São Paulo/Laboratório Nacional de Astrofísica in Brazil.

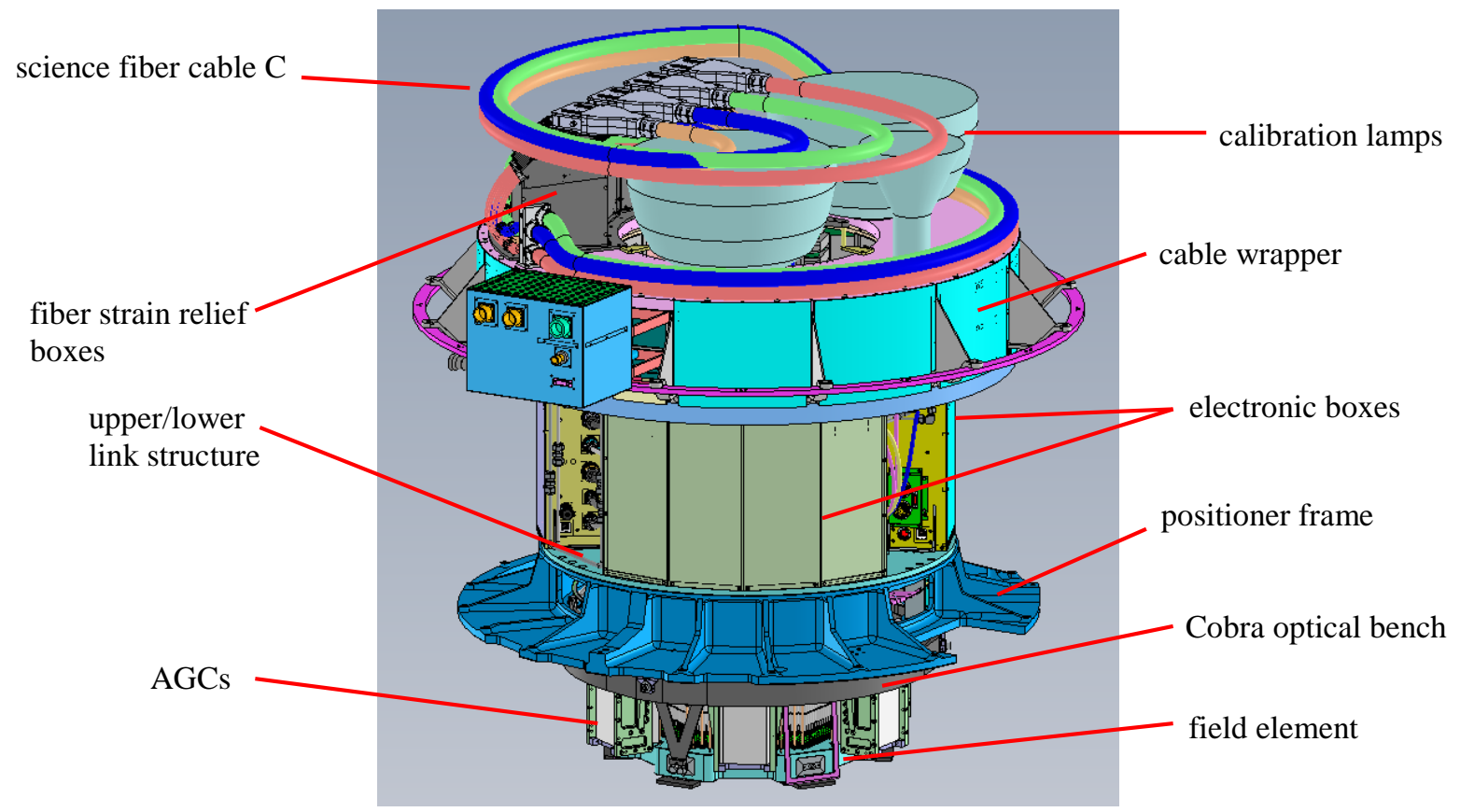

Figure 1. The components of PFI. The top cover for the storage of science fibers was removed to show the structure inside.

\section{PFI STRUCTURE INTEGRATION}

The basic design requirements and details of the PFI components were presented in 2014 and 2016 SPIE meeting ${ }^{3,4}$. Figure 1 shows the schematics of the PFI with major components indicated. Most components follow the original design and have been manufactured accordingly. The PFI integration started from the integration of the positioner frame (PF) with the COB. The fiducial fibers were installed after this as the process requires enough space for the handling and routing of the fibers. The installation of the upper lower link structure and the cable wrapper followed. With the main stricture integrated, the PFI cables and coolant lines were assembled. Then, the electronics boxes were installed. After the cables and coolant lines were checked, the installation of the Cobra positioner modules began. All integration processes were completed on a test stand so that we could measure, calibrate, and test the components. Throughout the process, various tests were executed 
to confirm the system performance including the focal plane location, the rotation torque of the cable wrapper, the power, network and telemetry signals and the pressure test of the coolant lines.

\subsection{The location and deformation of the PFI focal plane}

The focal plane location of PFI is critical to the throughput and performance of the PFS. It is mechanically defined by the distance from the PF reference surface, which interfaces with the telescope structure, to the surface of the fiber microlens. As the length of the Cobra positioners and fiber assembly is fixed, this distance can only be adjusted by the shims between the PF and the bipods of the COB. From the optimized location of the PFI focal plane, the distance from the reference surface of the PF to the bottom surface of the COB should be $93.359+/-0.05 \mathrm{~mm}$ at $20{ }^{\circ} \mathrm{C}$. This distance is based on the measured length of Cobra $124.57+/-0.015 \mathrm{~mm}$. Figure 2 shows a picture of the integrated PF and COB structure. The two pieces are linked with the three bipods.

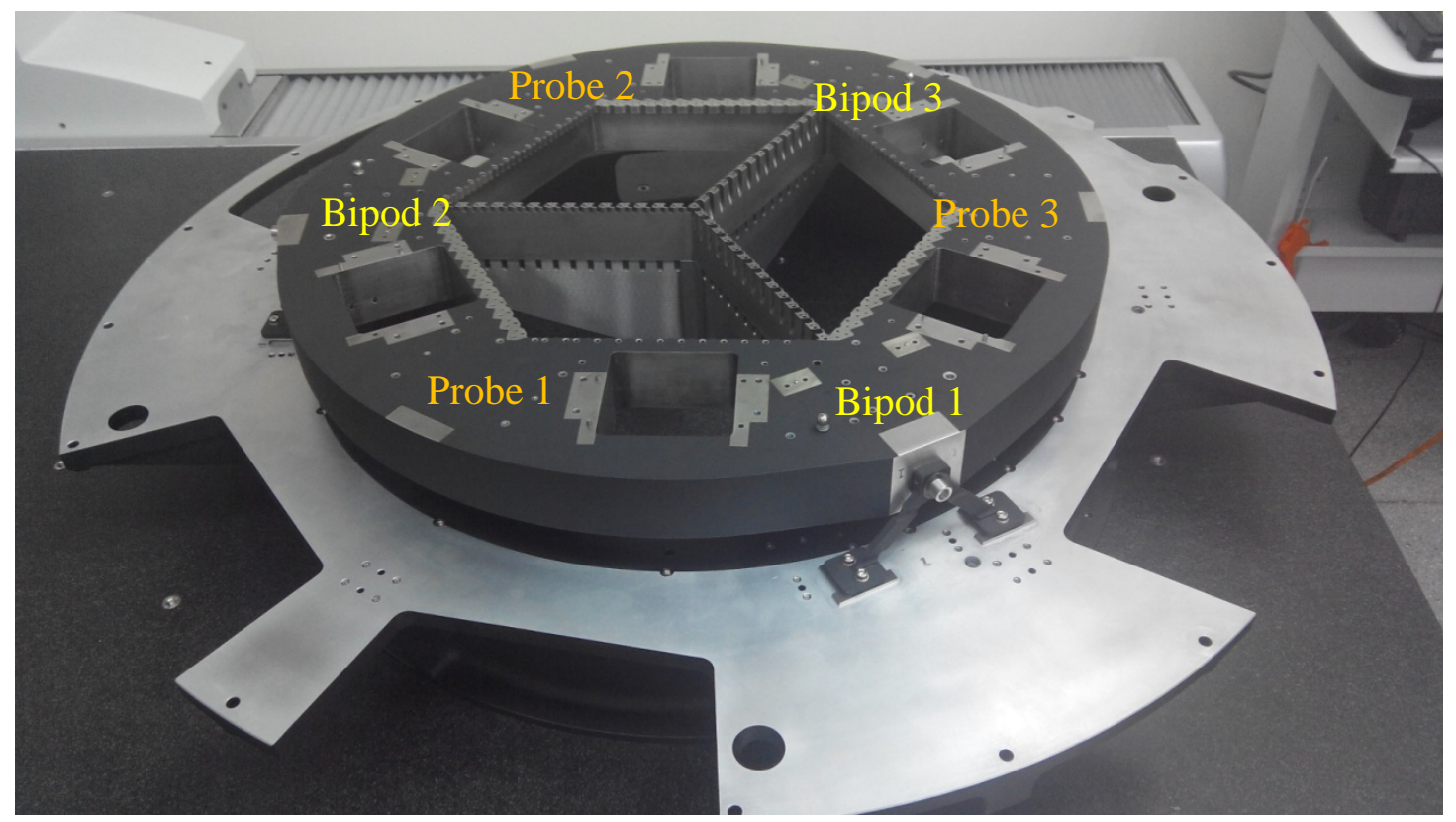

Figure 2. The integrated PF with COB. The bipods connecting the COB and the PF control the height. In order to show the structure clearly, the picture was taken upside down. The distance measurement was done with the normal gravity direction.

The distance was measured by a FASO laser tracker. We picked 5 points to set up a reference plane at position frame of each bipod fixed position, and then picked 3 point on COB surface to calculate the vertical distance of each point to reference plane. After a careful shimming process, the distance at three bipods are $93.367 \mathrm{~mm}, 93.363 \mathrm{~mm}$ and $93.367 \mathrm{~mm}$, respectively. The measurement error is about $5 \mu \mathrm{m}$. The distance is within $10 \mu \mathrm{m}$ to the nominal distance and the difference among the three bipods are within $5 \mu \mathrm{m}$.

After the shimming, we measured the deformation of the structure. The full dummy weight of PFI was loaded including $80 \mathrm{Kg}$ load at the center and $45 \mathrm{Kg}$ at the field edge for the weight of AGCs and the field element. The displacement was measured by three Keyence laser displacement probes installed at the three corner of the COB. The locations of the probe were also shown in figure 2. At 90 degrees elevation, the measured deflection after adding all dummy weight is 33 , 39 and $38 \mu \mathrm{m}$ toward the gravity direction. The number is from the average of 2 different cycles of dummy weight installation and the differences between the 2 measurements is within $4 \mu \mathrm{m}$. Using this structure, the deflection at 30 and 60 degrees of elevation angles was also measured. Table 1 shows the average result of three measurement. Positive number means the relative move away from the PF. The differences between the 3 measurements is $1 \mu \mathrm{m}$. When the PFI is tilted in the $-\mathrm{Y}$ direction, the associated probe 1 location on the PF is the highest points (see figure 3). This makes the probe 1 move further away from the PF while the probe 2 and 3 location move toward the PF as expected.

Combing the deflection at 90 degree, the overall deflection at different elevation angle is in the following table. The three numbers in each cell shows the deformation measured by the three probes. As expected, the probe 1 location has the largest 
deflection and the number can be higher than $50 \mu \mathrm{m}$ at 30 degree elevation. This level of deformation is consistent with the theoretical structure analysis done back in the CDR phase. The measurement results confirm the integration of the mechanical structure deformation is repeatable and it is within the design requirements.

Table 1. The measurement items and accuracy for PFI.

\begin{tabular}{|c|c|c|}
\hline 90 degree elevation & $\mathbf{6 0}$ degree elevation & 30 degree elevation \\
\hline $33,39,38(\mu \mathrm{m})$ & $49,26,21(\mu \mathrm{m})$ & $58,8,-1(\mu \mathrm{m})$ \\
\hline
\end{tabular}

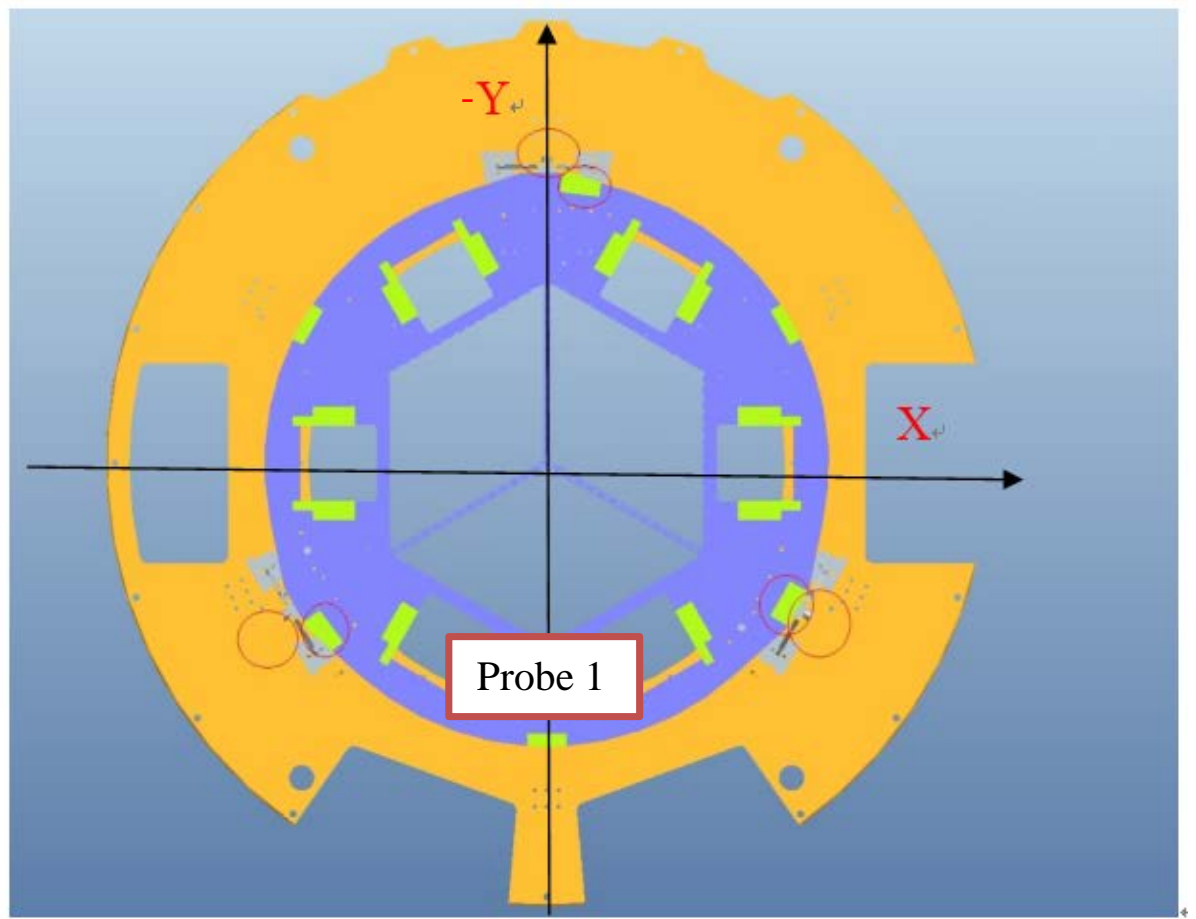

Figure 3. The rotation axis (x axis) of the $\mathrm{PF}+\mathrm{COB}$ structure and the location of probe 1 . The tilt is toward the $-\mathrm{Y}$ direction.

\section{THE TEST OF COBRA MODULES}

The 2394 Cobra positioners of PFI are integrated as linear modules to facilitate the characterization and integration process. 42 modules, each of which is populated with 57 Cobra fiber positioners integrated with fiber optics, populate the whole PFI focal plane. Figure 4 shows the model of a Cobra module. In each module, the Cobras are aligned in two rows; one with 29 Cobra positioners and the other one with 28 Cobra positioners. The details of Cobra actuator can be found in the SPIE proceeding ${ }^{5}$. For each Cobra module, the geometry of each Cobra positioner is essential for the future operation to move fibers to the target positions. A Cobra module measurement system was setup to measure the geometry of the Cobras including the arm lengths, the center of each stage and the hard limit positions for the two stages. Figure 5 shows details of a Cobra motor. With the measured geometry parameters, the convergence to targets of all Cobras positioners could be also tested.

The test bench consists of driving electronics, a supporting stand for the Cobra module, a light source and two video cameras. Figure 6 shows pictures of the setup. During the test, the fibers were back illuminated so that the camera can detect the spots of the fibers on the positioner side. The key element for the test is the video camera which records the positions of the fibers moved by the positioners. We used two cameras to cover the whole Cobra module. By analyzing the images, the fiber locations and thus the positioner movement can be obtained. The pixel size of the camera is $4.8 \mu \mathrm{m}$ 
with a format of $2592 \times 2048$ Pixels. Before the real test started, we calibrated the scale of the image and one pixel of the image corresponds to $82.5 \mu \mathrm{m}$ in the real space.

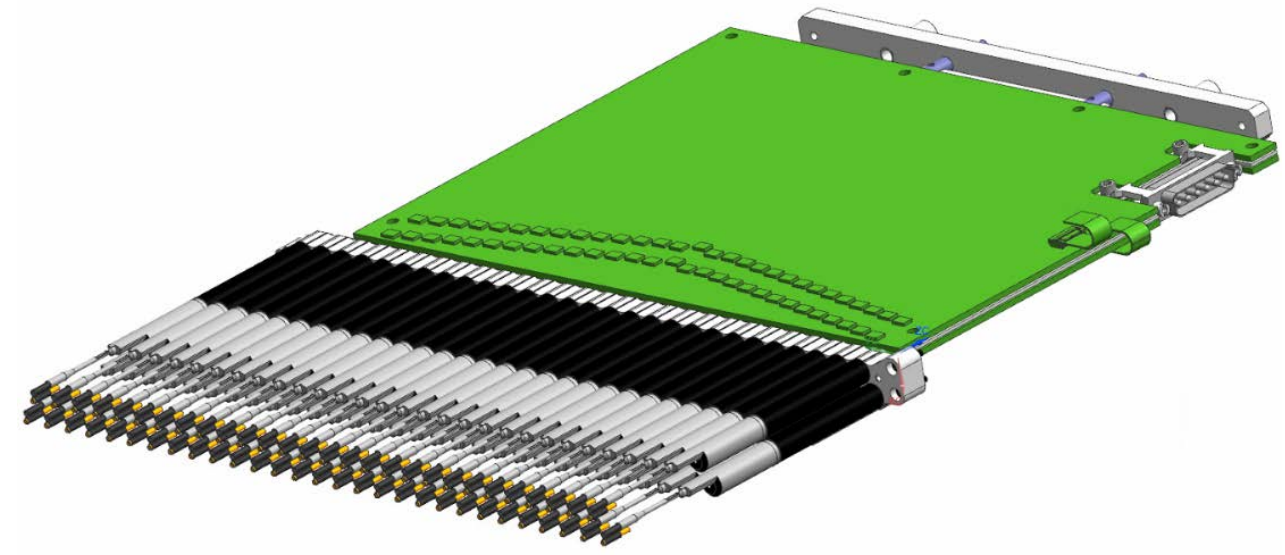

Figure 4. A 3D model of a Cobra module. The size is $30 \mathrm{~cm} \times 24 \mathrm{~cm} \times 1.5 \mathrm{~cm}$, and the weight is $1 \mathrm{~kg}$.

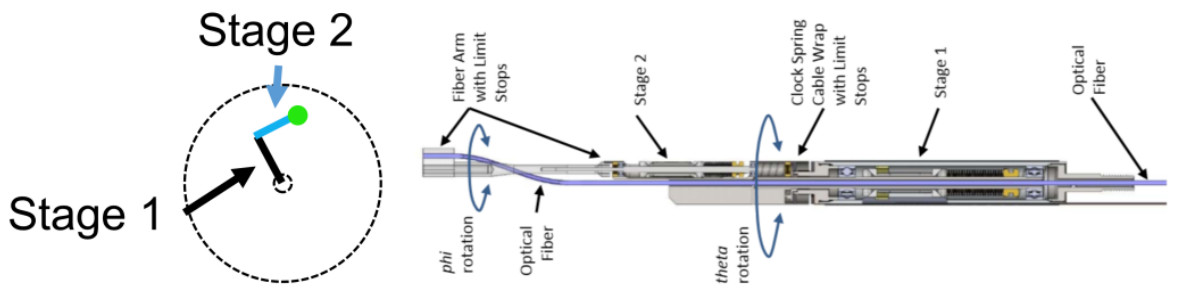

Figure 5. Details of Cobra fiber positioner. Stage 1 is called “Theta”, and Stage 2 is called "Phi”. The radius of theta/phi rotation is called "arm length". The range of motion is represented by how much extent the theta/phi rotation goes in degree.

During the measurement, we commanded the Cobra positioners to make circular motion stage by stage so that the cameras can take images along the circles generated by each motor stage. Combining the centroids from all images, we fit the arcs (in the case of Phi stage) and circles (in the case of Theta stage) of the Cobra positioner motion to estimate the centers and radius of the movement. Figure 6 are examples of the stacked images taken during the circular motion. The images were used to calculate the center, the radius (arm length) and the hard limit angles for the motors. To calculate the Theta arm length, we used the centers of Phi stage and the Theta stage. The Theta stage arm length is the distance between the two centers. In the image, we could also found that the density of the spots differs at different angle. Since the video camera took images with a constant interval, the variation of the spot density shows the variation of angular speed of the motors at different angles. These images were used to calculate the so called "motor map" which means the angular speed of a motor at different angles.
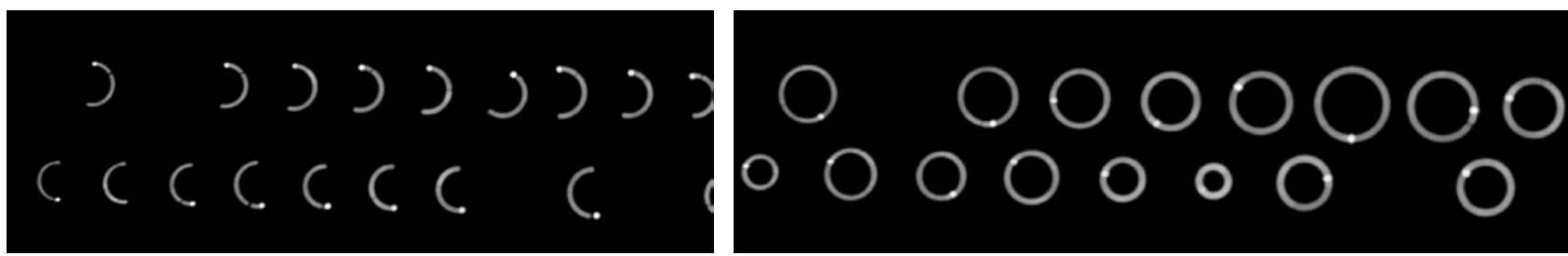

Figure 6. The stacked images of the Phi stage movement (left) and the Theta stage movement (right) for measuring the arm length and the motor center. There are few missing spots due to the broken fibers of the Spare \#1 Cobra module. 


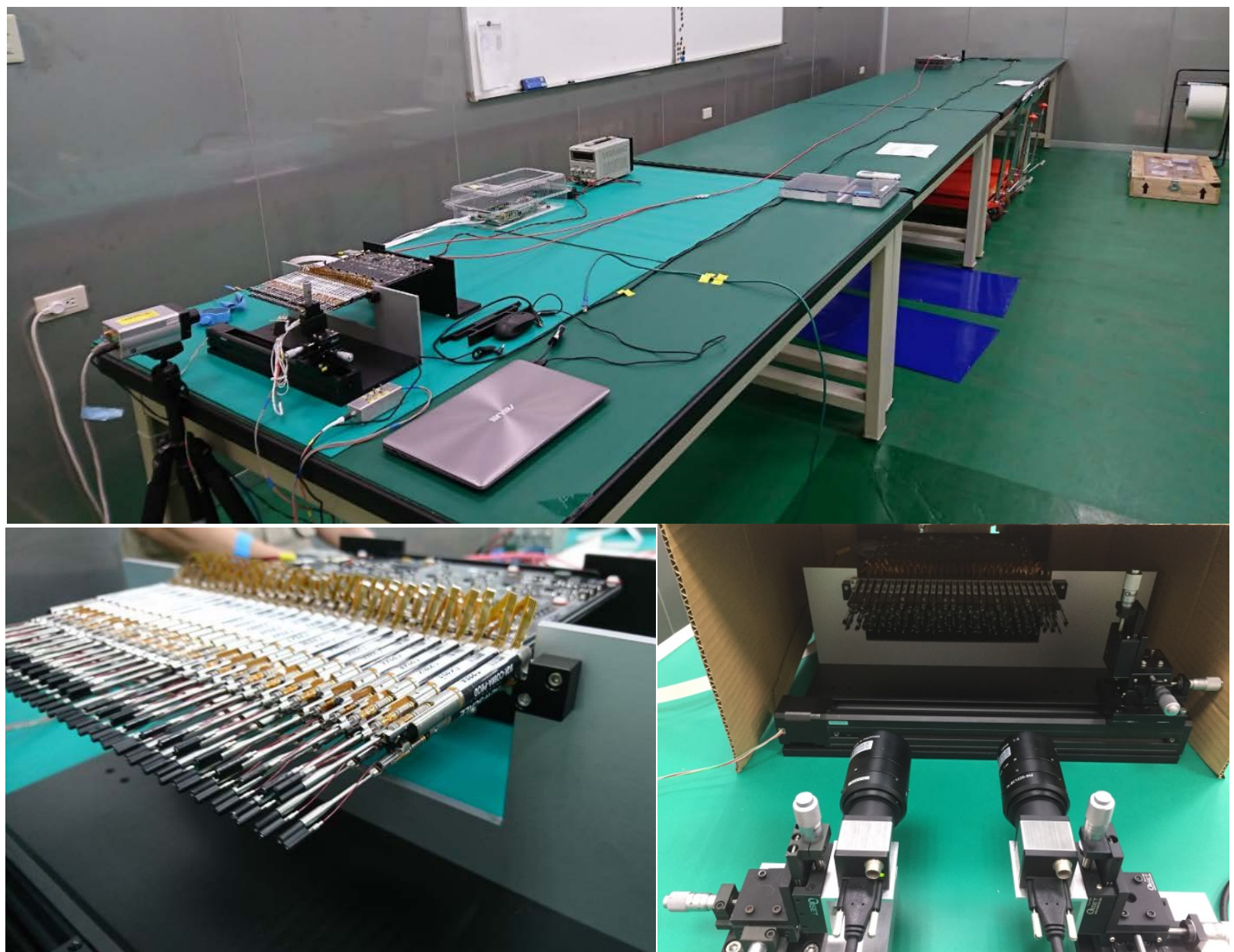

Figure 7. The Cobra module on the test bench in our lab (top). The Spare \#1 Cobra module on the support stand (bottom left) and the video cameras for the fiber position measurements (bottom right).

To generate motor maps, we command the Theta and Phi stages separately to make the corresponding circular move. Each time, we start the motion from counter clockwise hardstops and move the motors for 250 steps and calculate the angle associated with the motor movements. We continue the 250 steps moves until the motors hit the clockwise hardstops. With the angle differences of each move, we can generate motor maps with motor speed at different angles for the forward move. Then, we move the Cobras backwards with also 250 steps to generate motor maps for the reverse move until they reach the counter clockwise hardstops. The measurement is repeated for three times to generate average motor maps. Figure 8 shows the motor map of the two stages of positioner ID \#11 for Cobra module \#10. Typically, the motor speed could vary significantly over the whole range of motion as shown in the plot.

The Cobra motor map depends heavily on the "on time" value. On time is the duration of the Cobra driving signal pulse which can be adjusted roughly from 10 to $160 \mu \mathrm{s}$. Basically, the motor speed increases with the on time. The motor speed is critical to the precision of the Cobra positioner movement but a slower motor speed will increase the overhead to reach the target positions. Based on the required precision to reach the targets within $10 \mu \mathrm{m}$, the ideal motor speed is about 0.06 degree/step. We preselected two on time to get the linear relationship between the motor map and the on time. With the linear relationship, the on time to reach the target motor speed can be calculated. In the process, typically we adjust on time for 4 times to reach the target speed. It should be noted that for most Cobra positioners, it is straight forward to adjust the motor speed. However, there are always few outliers that show non repeatable results and it is not easy to adjust the motor speed to the optimized value. Figure 9 shows the histogram of the average motor speed for the Phi and Theta motors after the on time adjustment for science module \#10. It is clear that most on time adjustment were successful and the Cobra motors can move around 0.06 degree/step. 

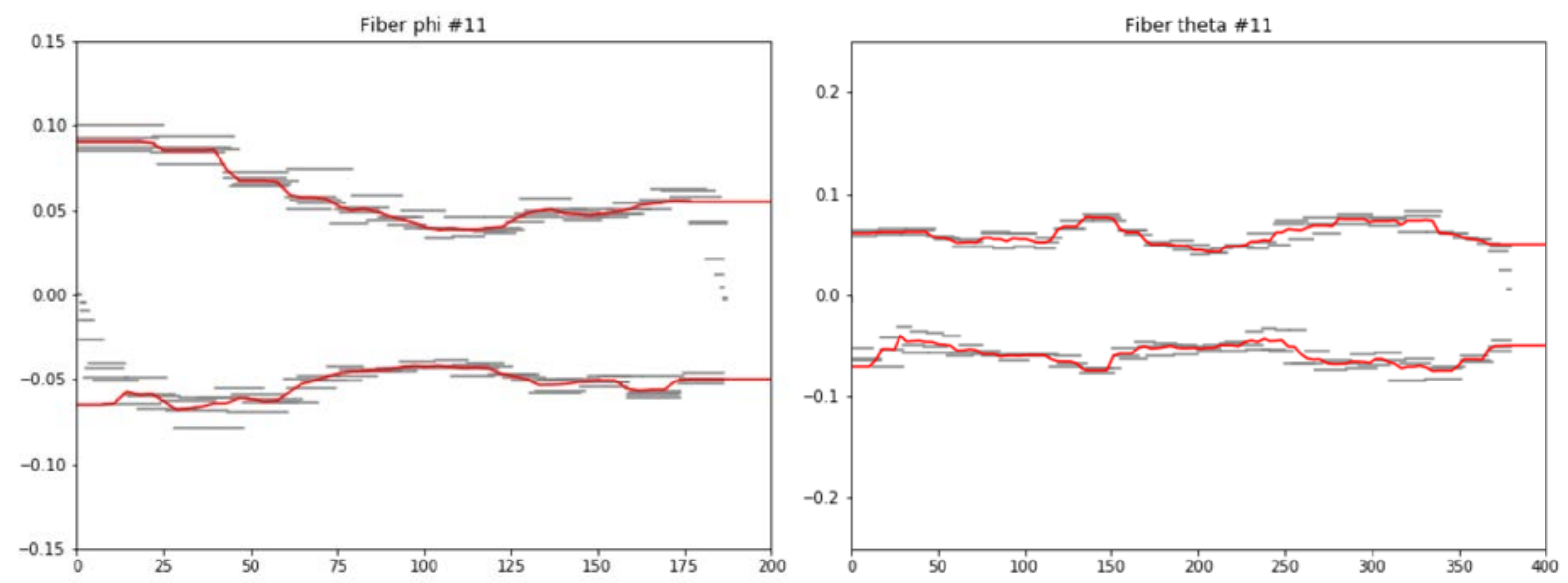

Figure 8. The motor map for the Phi motor (left) and Theta motor (right) for Cobra \#11 of module \#10.
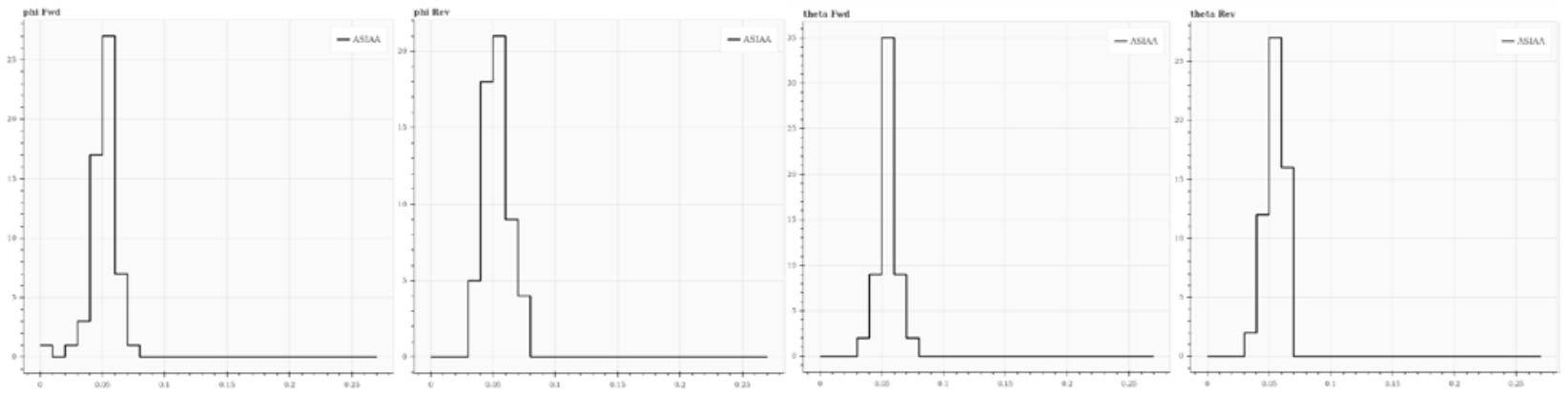

Figure 9. The histogram of average speed for Phi and Theta motors of module \#10 after the on time adjustment.

With the geometry parameters and the optimized motor maps, we commend the Cobra to move to assigned angles with the target convergence software. After each Cobra positioner move, we use the camera image to calculate the Cobra locations and the distance to the targets to decide the steps needed for the next move. The iteration continues for 8 times and we check the final angular distance between the final Cobra positions to the target positions. In order to avoid the unwanted collisions between the Cobra positioners, for the module tests, we only carried out the one dimensional tests, meaning testing one stage of the motors at one time. For Phi motors, we selected targets angles from 15 degrees to 165 degrees respected to the home position with 10 degrees separation, in total 16 target positions. For Theta motors, since the range is larger, the target angles are from 15 degrees to 345 degrees with 10 degrees separation, in total 34 target positions. We tried to avoid the angles close to the hardstops to reduce the risk of hitting hardstops too many times. Also, to avoid the collisions between the Cobras, the two rows of Theta motors were positioned away from each other, i.e. vertical to the rail during the Phi target convergence. Phi motors were at 60 degrees position while we had the Theta target convergence tests.

Considering the possible error and the instability for the Cobra positioner movement, during the target convergence iterations, we monitor the actual angle moved for each iteration and compare with the expected angular movement to adjust the on time of the motors for the next move to ensure the Cobra positioners can move with the expected speed and converge to the target position. Figure 10 shows the target convergence result of Theta motor \#11 of the Cobra module \#10. As shown in figure 10, the error between the targets and the fibers is usually large for the first two moves. After that, the Cobra positioners can quickly reach the targets within 1 degree in the 3rd or 4th iterations in most cases. 

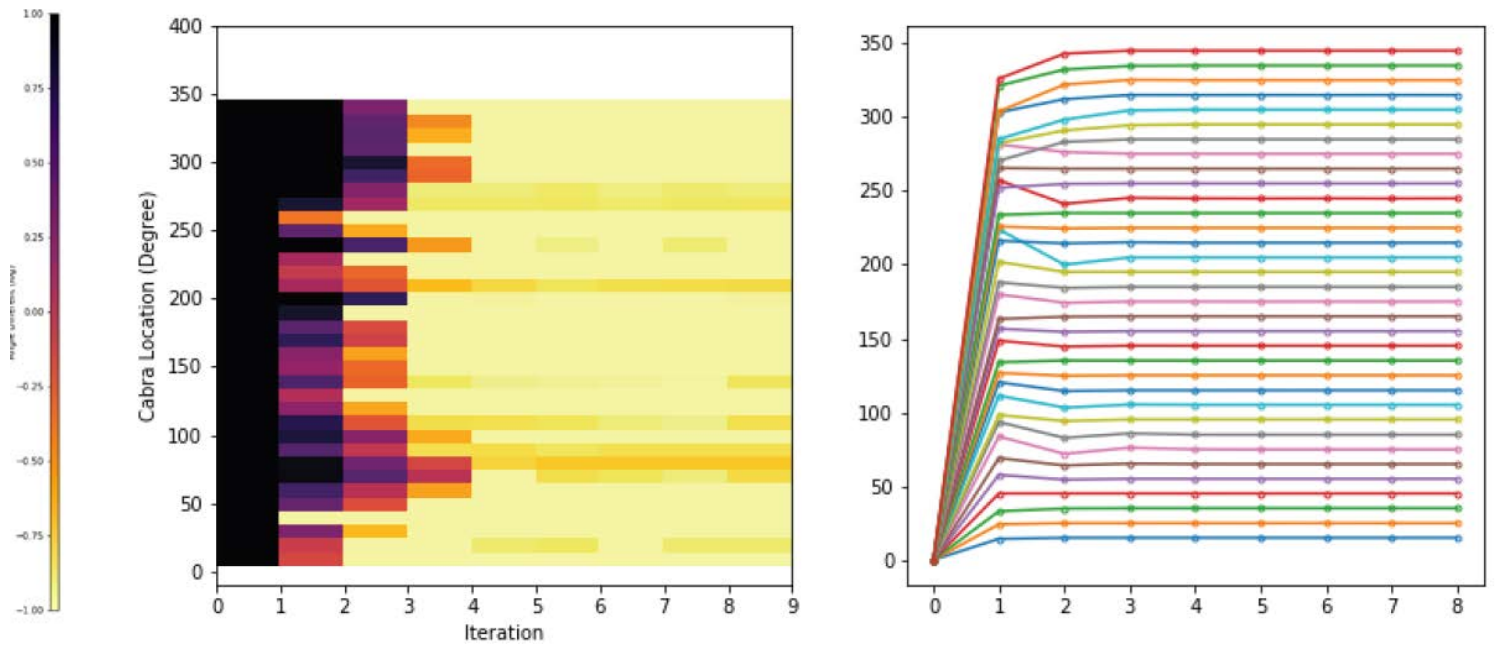

Figure 10. The target convergence result for the Theta motor of Cobra \#11 of module \#10. The left panel shows the angle different between the motor and the target and the right panel shows the location of the motor at different iterations. The horizontal axis is the iteration number and the vertical axis is the target angle.

In order to quantify the performance for target convergence, we defined the signal to noise ratio (SNR) for each Cobra positioner after each iteration. The SNR is defined based on the light coupling efficiency into the fiber and time needed to move the fiber to the required position, comparing with the typical exposure time. The equation used to calculate the SNR is:

$$
\mathrm{SNR}=\left[1-\left(\frac{d}{k_{\text {offset }}}\right)^{2}\right] \sqrt{\frac{t_{\text {max }}+t_{\text {obs }}-n \times t_{\text {step }}}{t_{\text {obs }}}}
$$

where $d$ is the distance to the target position, $k_{\text {offset }}$ is the coupling radius which is $0.075 \mathrm{~mm}, t_{o b s}$ is 900 sec; the typical integration time for PFS observations, $t_{\max }$ is $105 \mathrm{sec}$; the maximum allocated time to move the fiber and $t_{\text {step }}$ is $12 \mathrm{sec}$; the time used for each steps, $n$ is the iteration number. For simplification, we did not consider the light distribution of the objects. However, the SNR should still reflect the distance between the target and the fiber position. When fiber is within $10 \mu \mathrm{m}$ to the target, the SNR should be higher than 0.92 . The SNR is 0 if $d$ is larger than $0.075 \mathrm{~mm}$. Figure 11 shows the peak SNR plots of the 8 iterations for all Phi and Theta motors of the one dimensional target convergence of module \#10. Through the iterations, the SNR first increases when the fiber approaches the target but it decreases after fiber reaches the target.
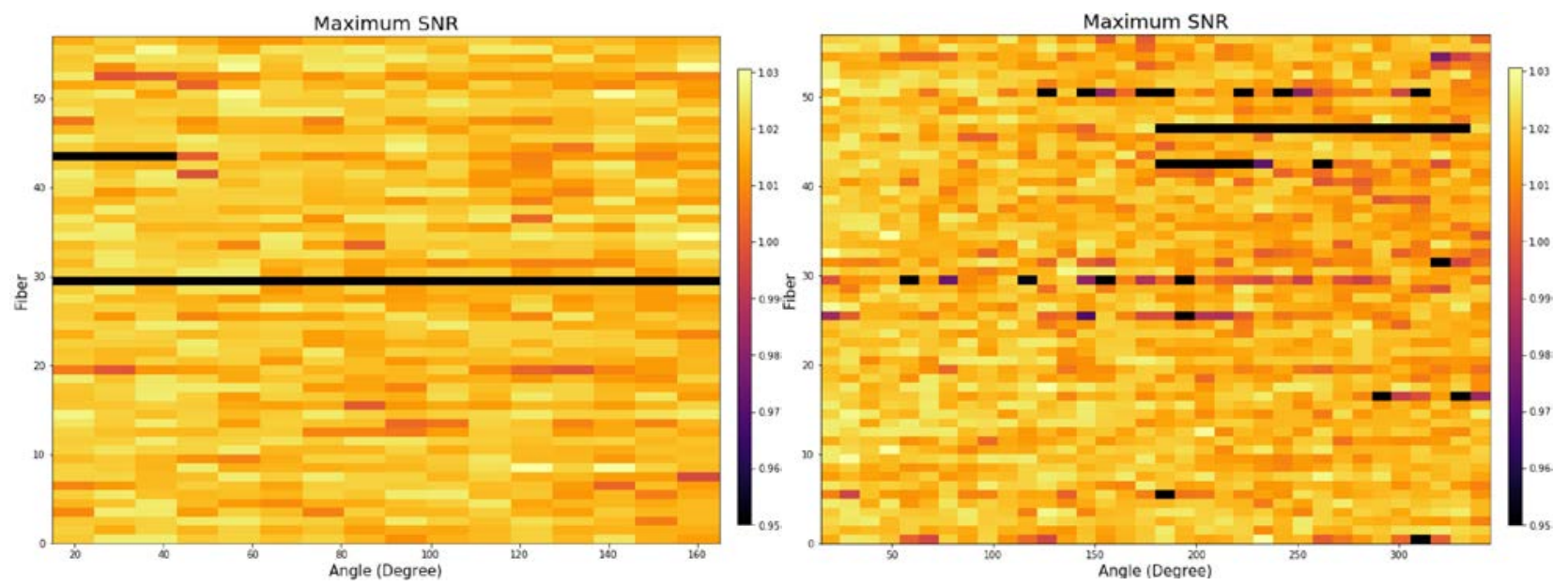

Figure 11. The peak SNR plots for all Phi motors (left) and Theta motors (right) of module \#10. The vertical axis is the Cobra positioner number in the module and the horizontal axis is the target angles. The color of each cell indicates the SNR of the particular target angle and positioner.. 
As shown in the plots, the Cobra can move to the target with SNR higher than 0.92 in most cases. The failed cases usually are originated from the unstable motor movement and the ratio of failed convergence cases is usually less than $1 \%$. For module \#10, the Phi motor \#10 was damaged so it always failed.

\section{COBRA MODULE INTEGRATION IN PFI}

After Cobra modules were tested and verified, we integrated the modules with the mechanical structure. As mentioned, we installed the fixed fiducial fibers before installing Cobra modules. As the modules are closely packed to each other with fiducial fibers in between, we added guiding plates and protection plates during the installation of Cobra modules to prevent any possible damage to fiducial fibers and installed science modules. Also, we designed a tool to safely handle Cobra modules throughout the process. The modules were installed from the bottom of PFI upwards and bolted on the COB. Figure 12 shows the pictures taken during the installation and the pictures of the guiding plates and the handling tool.

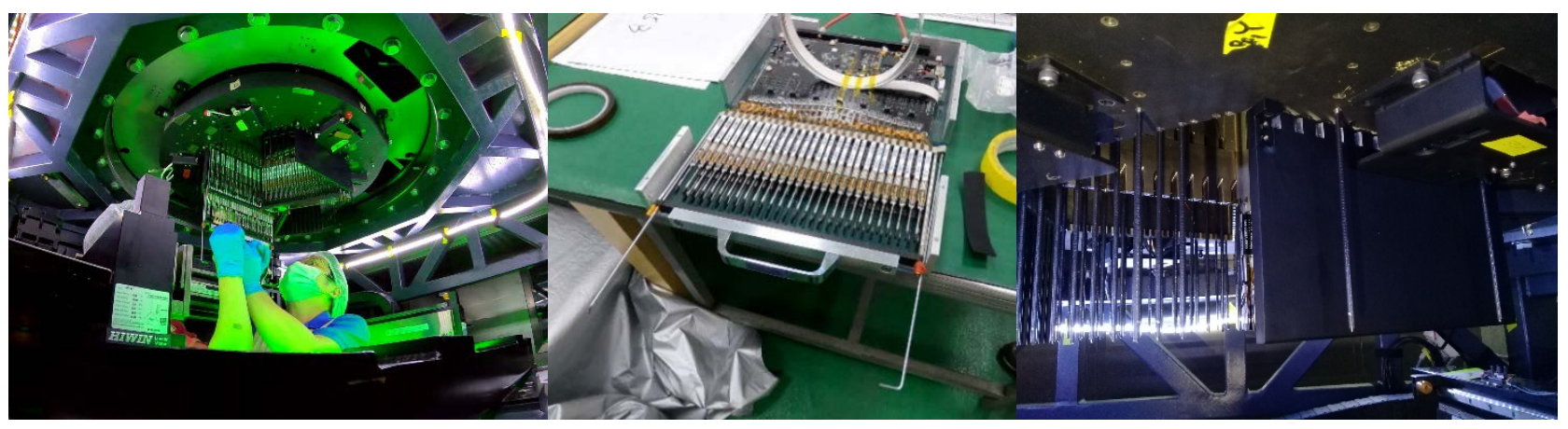

Figure 12. Left: the picture taken during the Cobra module installation. Middle: the handling tool of Cobra module. Right: the guiding plate for the Cobra module installation.

In order to reduce the risk of removing the installed modules, the Cobra modules were not installed at once but in 4 steps. We installed one module first, then added to 9 modules, 21 modules and finally a fully populated focal plane. In each step, we checked the Cobra performance and the control system. Then, we measured the home Cobra positions and also repeated the module test items to monitor if there is any change of the motor behavior with time. The Cobra home positions were measured with the fiber probe described in our SPIE 2016 paper $^{4}$ at different temperatures $\left(20,5,0\right.$ and $\left.-5{ }^{\circ} \mathrm{C}\right)$ and different elevation angles (90, 60 and 30 degrees) to understand the Cobra positioner deformation during exposures. Since we already know the mechanical structure deforms at different conditions, we used the fiducial fibers as the references to define the relative shift of the Cobra positioners. In real operations, we also use the metrology camera to image the fibers so fiducial fiber locations are essential references to the calibration of the science fiber locations. Figure 13 show the difference of the Cobra home positions between 90 degrees to 30 degrees of elevation angle at $20^{\circ} \mathrm{C}$.
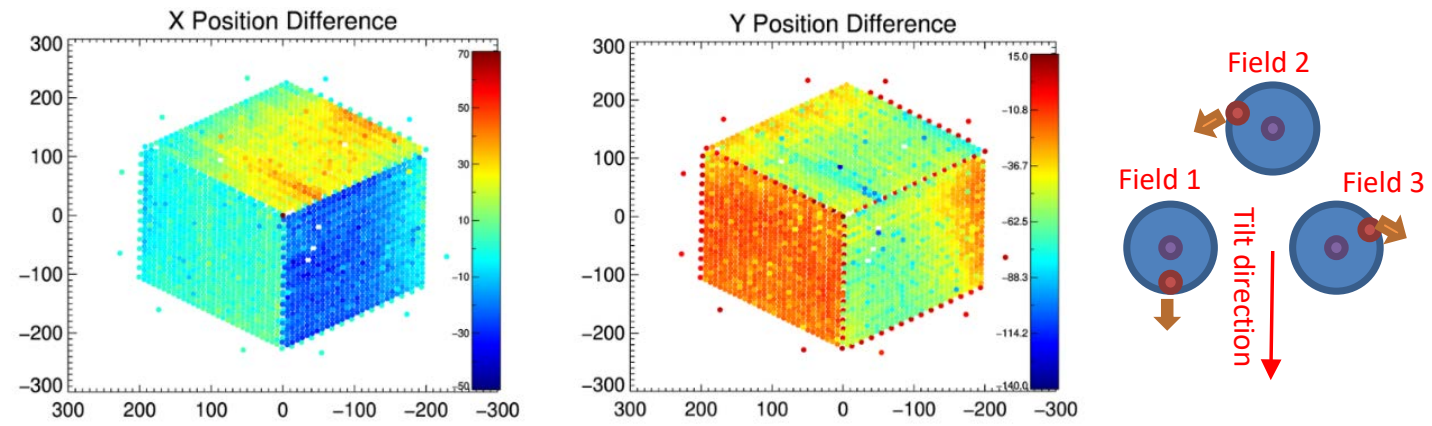

Figure 13. The difference of the Cobra home positions in X and Y direction between 90 degrees to 30 degrees of elevation angle at $20{ }^{\circ} \mathrm{C}$. The color bar shows the scale in the unit of $\mu \mathrm{m}$. The inset on the right side shows the deformation direction of the positioners in different fields. 
When the elevation changes (tilt in -Y direction), it is clear that the Cobra positioners at different parallelogram (called field) showed different deformation. We found the deformation direction is related to the orientation of the Theta motor. In our test, the Theta motor orientation was parallel to the rail of the module and pointed outward from the field center as shown as the red spots in the inset of figure 12. The result shows that in field 1 , the deformation is along the -Y direction, i.e. the gravity direction. In field 2, and 3, the deformation direction is tangential direction, i.e. the moving direction, of the Theta motor movement as shown as the arrows in figure 12. The deformation is larger for Cobra positioners in field 2 and 3 since the deformation direction is in the moving direction of the Theta motors. In general, the maximum deformation is around $30 \mu \mathrm{m}$ for 60 degrees of elevation change and we expect $<5 \mu \mathrm{m}$ deformation during one exposure time.
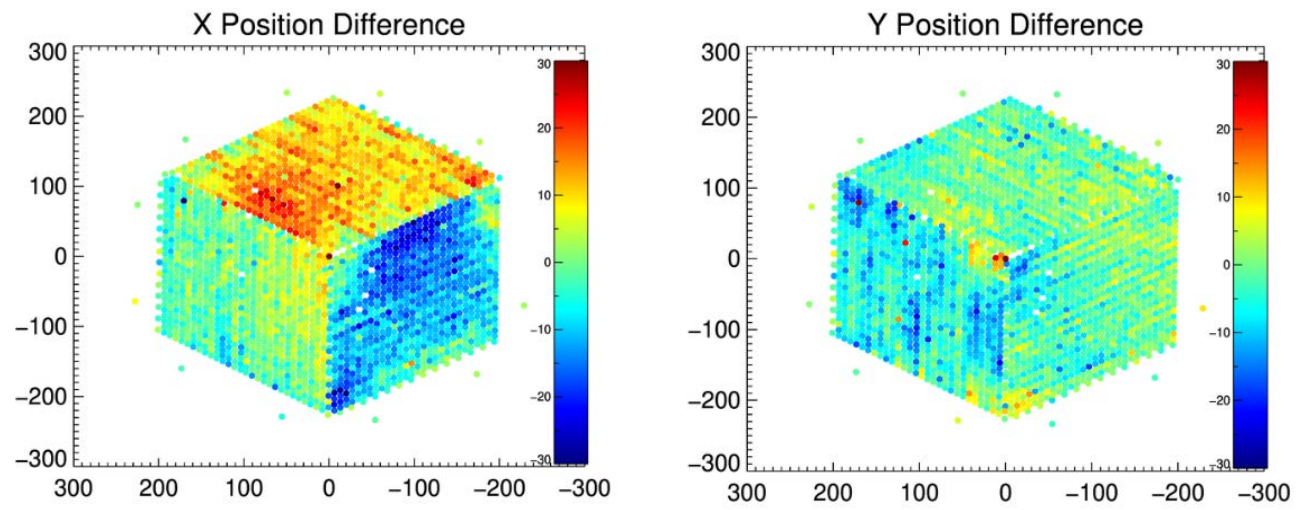

Figure 14. The difference of the Cobra home positions in $\mathrm{X}$ and $\mathrm{Y}$ direction between 5 to $-5{ }^{\circ} \mathrm{C}$ at 90 degrees of elevation angle. The color bar shows the scale in the unit of $\mu \mathrm{m}$.

For the deformation associated with temperature change, we found a quite different behavior compared with the elevation change. Instead of clear different among the three fields, we saw the linear pattern in all 3 fields meaning different deformation coming from the Cobra positioners on the two rows of each module. After some investigation, we concluded that this is caused by the aluminum fin which holds the control boards of the Cobra module. The aluminum fin is glued in the middle of the Invar rail. The difference of thermal expansion coefficient between Aluminum and Invar rail causes the bending of the rail slightly and the two rows of the Cobras tilted to generate the deformation we measured. This generates around $20 \mu \mathrm{m}$ of shift from 5 to $-5{ }^{\circ} \mathrm{C}$ temperature change. It should not generate too much shift during the 15 minutes exposures given the temperature change should not be dramatic.
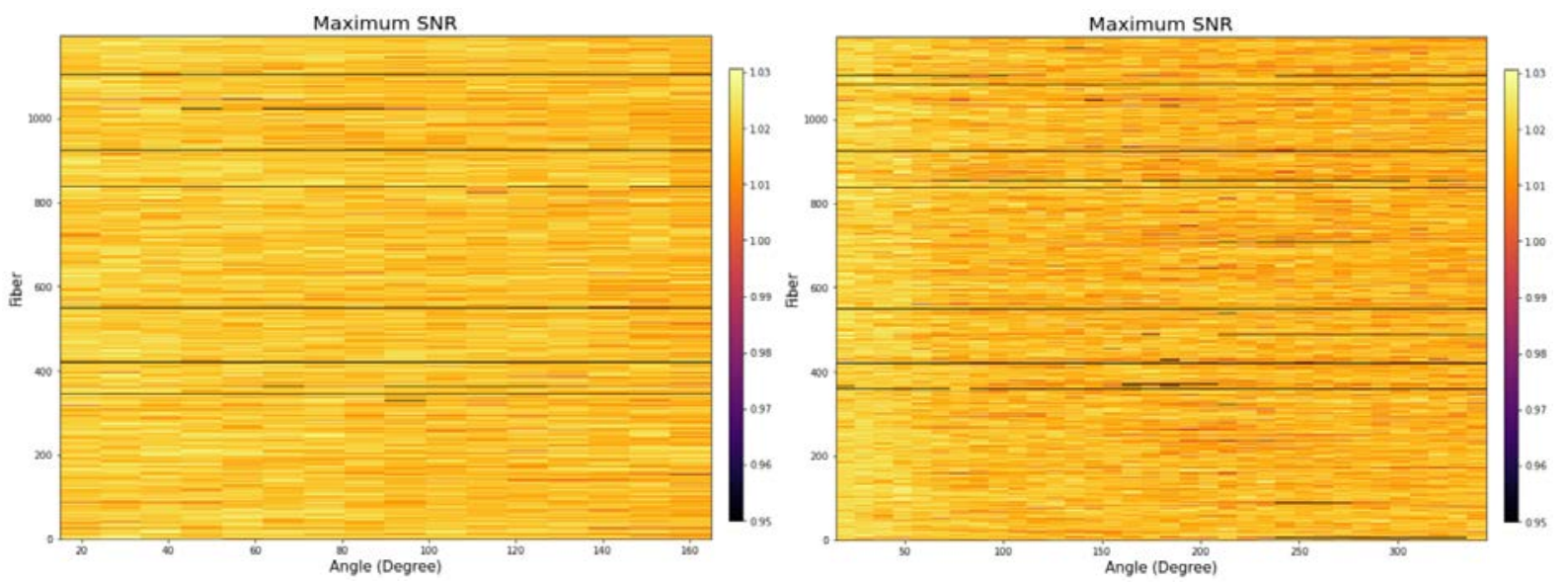

Figure 15. The peak SNR plots for the target convergence tests for 21 modules installed. The left panel is the plot for all Phi motors and the right panel is the plot for all Theta motors. The vertical axis is the sequential Cobra positioner number and the horizontal axis is the target angles. The color of each cell indicates the SNR of the particular target and Cobra positioner. 
After the home measurements, we also tested the one dimensional target convergence performance after the Cobra modules were installed. Figure 14 shows the peak SNR plots of the target convergence tests with 21 module installed. As shown in the figure, most Cobra positioners can reach the target. The failure rate is about $0.13 \%$ for all Phi motors and $0.27 \%$ for all Theta motors. This give us a high confidence of the final performance of the PFI. The black lines indicate the Cobra with either broken fiber or motor. Figure 15 shows the focal plane after all modules were installed.

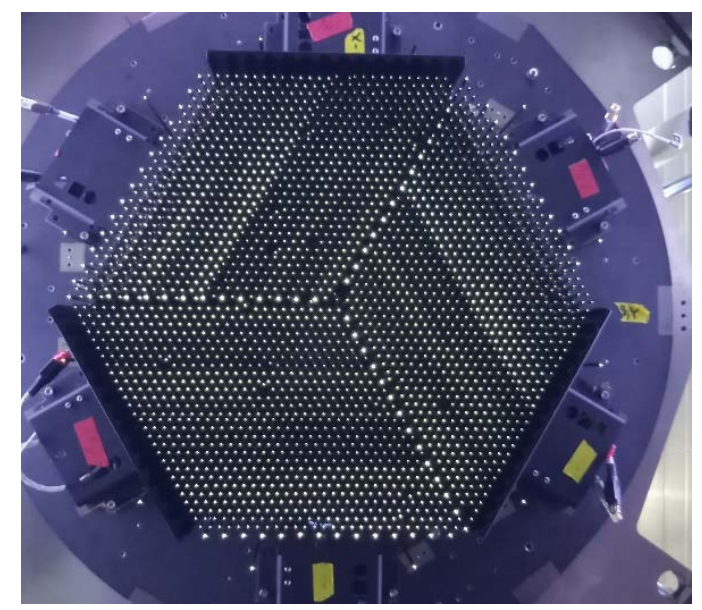

Figure 16. The fully populated PFI focal plane with back illuminators on. The broken fibers could be identified from the missing spots in the picture.

\section{CURRENT STATUS AND SUMMARY}

The integration and test of the PFI is still ongoing. After all Cobra modules were installed, there were 5 broken fibers in the system including 4 broken fibers damaged during the module fabrication. With all Cobra modules installed, we have started the connectorization of the Tower connectors each connects to one PFS spectrograph. The final target convergence tests with all modules will start in early 2021. We will also test the target convergence with the real target positions prepared for the survey. After it is completed, we will install the AGC and field element and the associated calibration and tests as the last step for the focal plane integration. Then, we will install the calibration lamp and the covers for the PFI. The PFI is planned to be shipped to Hawaii in May 2021.

\section{ACKNOWLEDGEMENT}

We gratefully acknowledge support from the Funding Program for World-Leading Innovative R\&D on Science and Technology(FIRST) "Subaru Measurements of Images and Redshifts (SuMIRe)", CSTP, Japan for PFS project. The work in ASIAA, Taiwan is supported by the Academia Sinica of Taiwan.

\section{REFERENCES}

[1] Sugai, H. et al. "Prime Focus Spectrograph for the Subaru telescope: massively multiplexed optical and nearinfrared fiber spectrograph", J. Astron. Telesc. Instrum. Syst. 1, 035001 (2015).

[2] Miyazaki, S. et al. "Hyper Suprime-Cam," Proc. SPIE. 8446, 84460Z (2014).

[3] Wang, S.-Y. et al. "Prime focus instrument of prime focus spectrograph for Subaru telescope," Proc. SPIE. 9147, 91475Q (2014). 
[4] Wang, S.-Y. et al. "The current status of prime focus instrument of Subaru prime focus spectrograph," Proc. SPIE 9908, 990882.

[5] Fisher, C. et. al. "Developing engineering model Cobra fiber positioners for the Subaru Telescope's prime focus spectrometer," Proc. SPIE 9151, 91511Y.

Proc. of SPIE Vol. 11447 114477V-12

Downloaded From: https://www.spiedigitallibrary.org/conference-proceedings-of-spie on 16 Dec 2020 Terms of Use: https://www.spiedigitallibrary.org/terms-of-use 